\title{
ICORMAN: Extended Version of CORMAN using Efficient Channel Reuse Method
}

\author{
Ashwini R. Jadhav \\ PVPIT, Pune, India
}

\author{
Navnath D. Kale \\ PVPIT, Pune, India
}

\begin{abstract}
Mobile Ad-hoc Networks (MANETs) are wireless networks in which all nodes are moving freely in the network; also they can leave or enter into the network at any time. The nodes in these types of networks are communicating on-the-move without any base stations [1]. Due to this mobility, inter-node connectivity may change frequently during normal operation which imposes new problems in network control, particularly in the design of higher level protocols such as routing. Routing is the most critical issue in MANET due to mobility of the nodes [2]. The performance of MANET is basically depends upon routing protocol use by the network. Routing protocol is nothing but the rules and regulations of routing which governs the journey of message packets from source to destination in a network. There were different routing protocols proposed for improving performance of MANET, such as DSR, DSDV, AODV, CORMAN, etc... All these protocols are best suitable in different network scenarios. However, among all these protocols, Ad-hoc on demand distance vector (AODV) is one of the protocols which are good for most of the network scenarios and it is the good solution for most of the network problems. But, AODV have some limitations too. It is not suitable for the opportunistic data transfer. Recently, the importance of utilizing the broadcast nature of multi-hop wireless networks is being recognized and considered in network protocol design, known as opportunistic data forwarding. One solution for opportunistic data transfer in MANET is CORMAN routing protocol. However, CORMAN does not give efficient performance during communication, because CORMAN does not make better channel reuse. By combining the better channel reuse method in CORMAN we can improve the performance of MANET. This paper focus on new proposed protocol called ICORMAN, the new protocol in MANET which makes the efficient channel reuse by using Ant colony optimization algorithm. Also better channel reuse method based on ACO is presented in this paper. The practical analysis of proposed work is possible using java in order to claim the efficiency of proposed routing protocol against the AODV \& CORMAN routing protocol which shows that, ICORMAN improves the performance of MANET as compared to AODV \& CORMAN routing protocols in terms of PDR, throughput, end to end delay and total PDR
\end{abstract}

\section{General Terms}

Mobile ad-hoc network, Routing Protocols, Protocol performance metrics

\section{Keywords}

AODV, CORMAN, Channel reuse, Ant colony optimization, Delay, Packet delivery ratio, Throughput.

\section{INTRODUCTION}

In the last couple of years, the use of wireless networks has become more and more popular. There exist three types of mobile wireless networks: infrastructure networks, ad-hoc networks and hybrid networks which combine infrastructure and ad-hoc aspects.

A mobile ad-hoc network lacks any infrastructure. There are no base stations, no fixed routers and no centralized administration. All nodes may move randomly and are connecting dynamically to each other [3, 4]. Simply, there is dynamic network topology which leads to frequent routing updates. And communication takes place via wireless means all nodes are operating as host as well as routers and are capable to discover and maintain routes to every other node in the network and propagate packets accordingly [5]. There are variable capacity links in MANET. The process of forwarding the packets from source to destination by using most efficient path is known as routing. Since there is dynamic topology in MANET [6], routing becomes the challenging issue in MANET. Therefore, research in wireless network was to make the wireless link as good as wired one and increase the performance. Unfortunately, this ignores broadcasting nature of wireless communication links. For mobile ad hoc networks to truly succeed beyond labs and testbeds, we must tame and utilize its broadcasting nature rather than fighting with it. A new research area namely, cooperative communication is an effective approach to achieving such a goal [7]. Research interest was increase in cooperative communication but more recently research interest is mainly focus on the top of the network protocol stack layers.

The performance of MANET is depends upon the used of routing protocols. There are various routing protocols present in MANET such as ZRP, DSR, DSDV, AODV, CORMAN etc. Among all, AODV routing protocol gives good performance for most of the network scenarios. However, opportunistic data transfer cannot be possible using AODV and therefore CORMAN was introduced to solve the problem of opportunistic data transfer in MANET [7, 8]. Using opportunistic data transfer CORMAN improves the performance of MANET, but CORMAN cannot make proper utilization of bandwidth and hence channel reuse. We can extend the CORMAN by using efficient channel reuse method based on ant colony optimization and the new version of CORMAN called as improved cooperative opportunistic data transfer in MANET (ICORMAN) which gives higher performance than both AODV \& CORMAN routing protocol.

\section{PROPOSED APPROACH FRAMEWORK AND DESIGN}

\subsection{Problem Definition}

The CORMAN is the cooperative opportunistic routing protocol in MANET [7]. It has good performance than 
AODV. But it does not make the proper bandwidth utilization and channel reuse. CORMAN could not find the optimal sequence of nodes for communication. And hence, the performance of MANET using CORMAN is an average only but not as good as necessary.

\subsection{Proposed Architecture}

The proposed protocol namely, ICORMAN makes the proper utilization of available bandwidth and makes efficient channel reuse and improves the routing performance of MANET in terms of PDR, delay, throughput and total PDR. For that ICORMAN uses ant colony optimization based algorithm. Following figure 1 indicates the architecture of proposed framework.

\subsubsection{ACO based Algorithm:}

The ACO_MH (Ant Colony Optimization Meta-Heuristic) is nothing but the group of methods those are inspired by 'foraging behavior of real ants' $[9,10]$. The original ants are starting from the start node and using the global as well as local knowledge in order to construct the shortest path towards destination node. The ACO based algorithms are responsible to finding out the optimal path from source mobile node to destination mobile node in MANET [11, 12]. In order to solve the problem of optimization using ACO based channel reuse method [13], the given problem is represented by using the graph theory. Using the graph, the sequence of nodes and its solution is constructed. In graph, the nodes are representing the problem components and links indicating the communication links between these components.

\section{Notations:}

$G^{\prime}=\left(V^{\prime}, E^{\prime}\right):$ Represents the problem

$\mathrm{V}^{\prime}=$ components of problem

$\mathrm{E}^{\prime}=$ communication links between components of problem.

\author{
Algorithm: \\ Step 1: the population of initial ants generated once input \\ communication paths discover.
}

Step 2: Optimization problem is build using the graph.

Step 3: Initial state assigned to every ant and finding out starting node for each ant.

Step 4: A 'probabilistic transition rule' is used by every ant in order to make a decision to move to the next node. This is based on the heuristic information and pheromone intensity.

Step5: A Heuristic function, nothing but the 'problem dependent function' for indicating the desirability of selected node.

Step 6: Pheromone intensity is build for representing the desirability of chosen path. This desirability of every path is discussed from the perspective of other ants.

Step 7: For pheromone intensity, the updating rule is used to finding the effect of the last deposited pheromones.

Step 8: The set of possible nodes $N_{i}^{k}$ is prepared for avoiding the forming a loop during the path construction. It shows the optimal nodes from the perspective of ant $k$ when it is placed on $i^{\text {th }}$ node.
Step 9: Finally the cost function is assigned to every selected path from source and destination node in order to show the optimal path of communication.

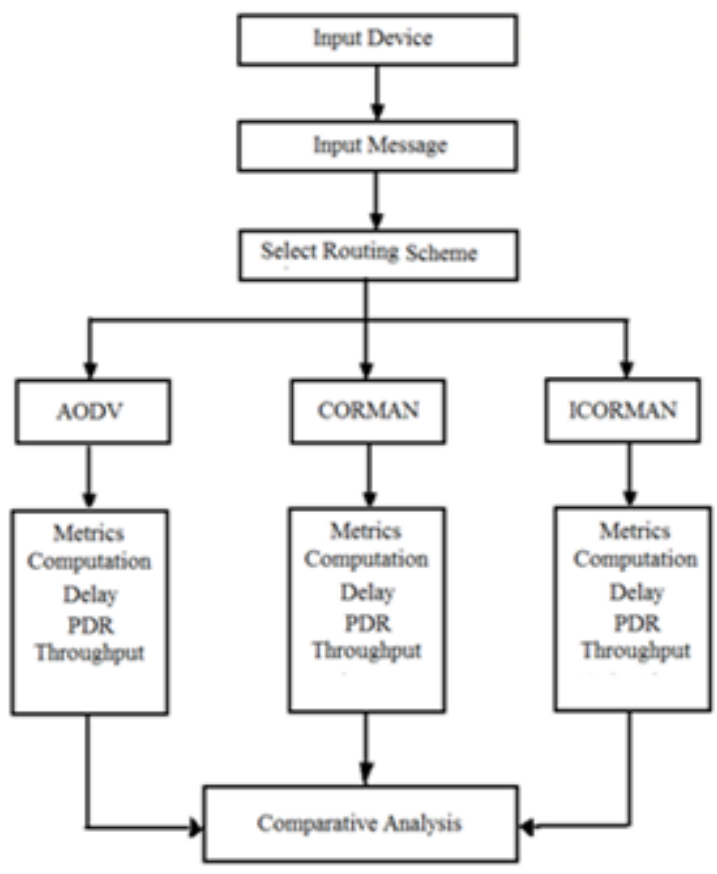

Figure 1: Proposed System Architecture

\section{IMPLEMENTATION}

Java can be used for implementation of proposed framework and to store the performance results of AODV, CORMAN and ICORMAN routing protocol MySql 5.4. The database keeps the performance records of all these protocols for packet delivery ratio, delay, and throughput. This system first take input devices to open those devices into the wireless network for communication. After opening the node next step is to allocate name to each node as an identification of that node. Then using any one node broadcast the message to every other node present in the network. Message passing is possible using three different protocols namely, AODV, CORMAN and ICORMAN routing protocol. When message is passed using AODV then message is trying to reach to every other node in the network. Sometimes it may happen that due to less efficiency of AODV routing protocol message may not be reach to particular node in the network. And sender node immediately shows the status of successful or unsuccessful communication with all nodes in the network. In this way, communication is also possible using other two protocols i.e. CORMAN and ICORMAN. After running all protocols, during communication performance of each routing protocol is stored into the database in terms of PDR, delay and throughput. From the database tables performance of those protocols can be observed and result indicates that ICORMAN routing protocol always shows higher performance than AODV as well as CORMAN routing protocol. And CORMAN performance is higher than AODV routing protocol in most of the network scenarios.

\section{PERFORMANCE ANALYSIS}

Following figures 2, 3, 4 and 5 respectively shows the protocol performances and comparative analysis of AODV, CORMAN and ICORMAN routing protocol. 


\subsection{End to end delay vs. network node.}

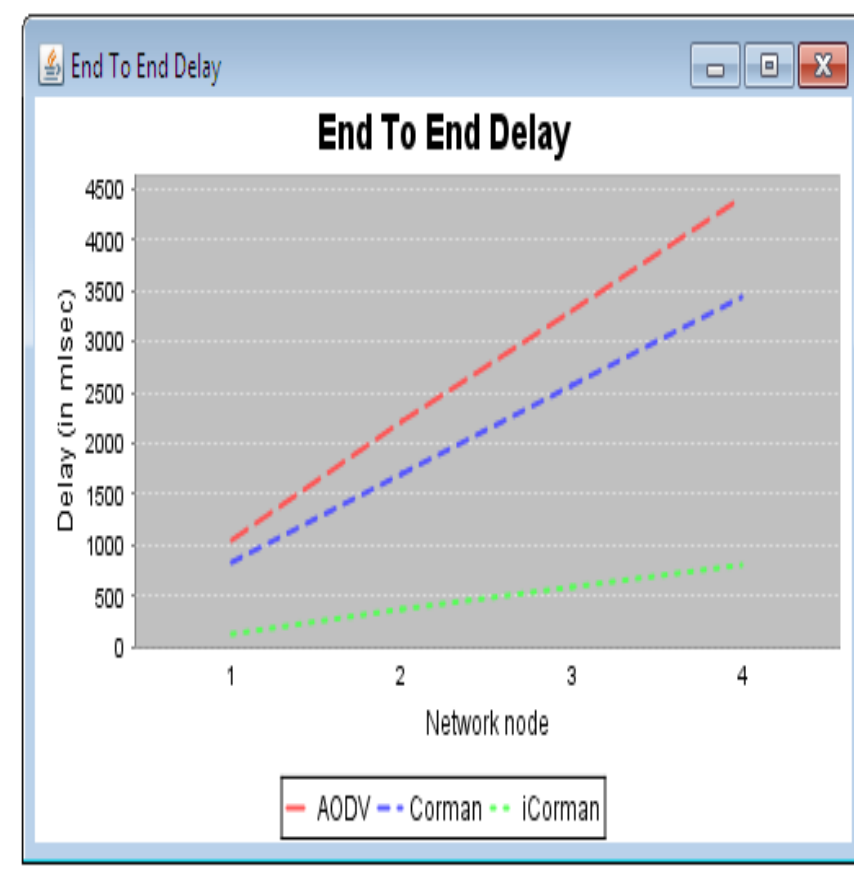

Figure 2: End to End Delay Vs. Network Node

Above figure 2 indicates end to end delay vs. network node. End to end delay is nothing but the total time required to transfer message from one node to another node. From graph it is cleared that AODV protocol needs $1050 \mathrm{mlsec}$ to transfer single message from first node to second node in the network. To reach the same message to third node it takes $2220 \mathrm{mlsec}$. The same message requires $3310 \mathrm{mlsec}$ to reach to node four and lastly it received to node fifth and for that purpose it takes $4410 \mathrm{mlsec}$. Above figure 2 also indicates end to end delay for CORMAN protocol. As shown above, CORMAN protocol takes $830 \mathrm{mlsec}$ to reach to node second. $1690 \mathrm{mlsec}$ are taken by CORMAN to transfer same message from node one to node third. To receive the same message from same node to node fourth CORMAN takes $2560 \mathrm{mlsec}$. And finally, same message reach to node fifth in $3450 \mathrm{mlsec}$ from same source node. Figure 2 also shows the ICORMAN performance for end to end delay. ICORMAN takes only $130 \mathrm{mlsec}$ from node one to node second. To transfer the same message from node one to node third it had taken $370 \mathrm{mlsec}$. The same message from same source taken $590 \mathrm{mlsec}$ to node fourth and finally that message also received to node fifth in $810 \mathrm{mlsec}$.

At the end it is observed that end to end delay for AODV routing protocol is higher, for CORMAN it is an average but for ICORMAN it less as compared to AODV as well as CORMAN routing protocols.

\subsection{Packet delivery ratio vs. network node}

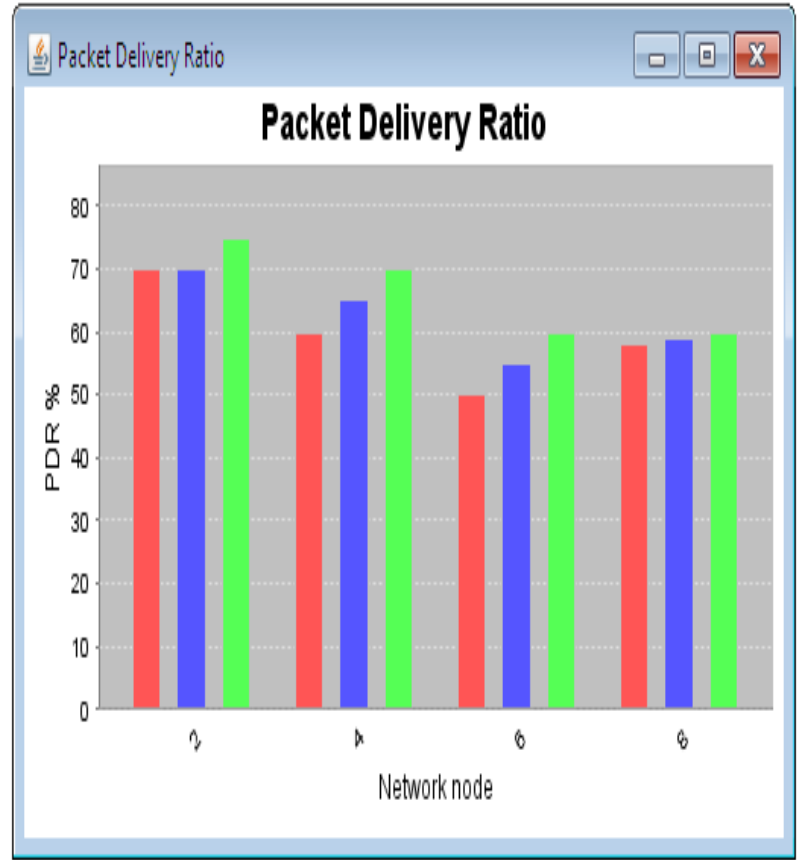

Figure 3: Packet delivery ratio vs. network node

Above figure 3 shows the packet delivery ratio vs. network node. In graph red color stands for AODV, blue for CORMAN and green for ICORMAN. PDR is nothing but the packet delivery ratio i.e. the percentages of delivered packets to each node with each protocol among three. For AODV routing protocol, PDR is $70 \%$ for second node in the network. For third node it is $60 \%$. Similarly, for fourth node PDR is $50 \%$ and for last node it is $58 \%$. When we observe the CORMAN routing protocol performance, we observed increase in performance like for second node it is $70 \%$ only, but for third node PDR is $65 \%$. For fourth node it is $55 \%$ and for the last node it is 59\%. ICORMAN shows 75\% PDR for second node, $70 \%$ PDR for third node, and for fourth and last node it is $60 \%$.

At the end it is observed that PDR is lower for AODV routing protocol. It is average for CORMAN routing protocol and higher for ICORMAN routing protocol. 


\subsection{Total packet delivery ratio vs. network node}

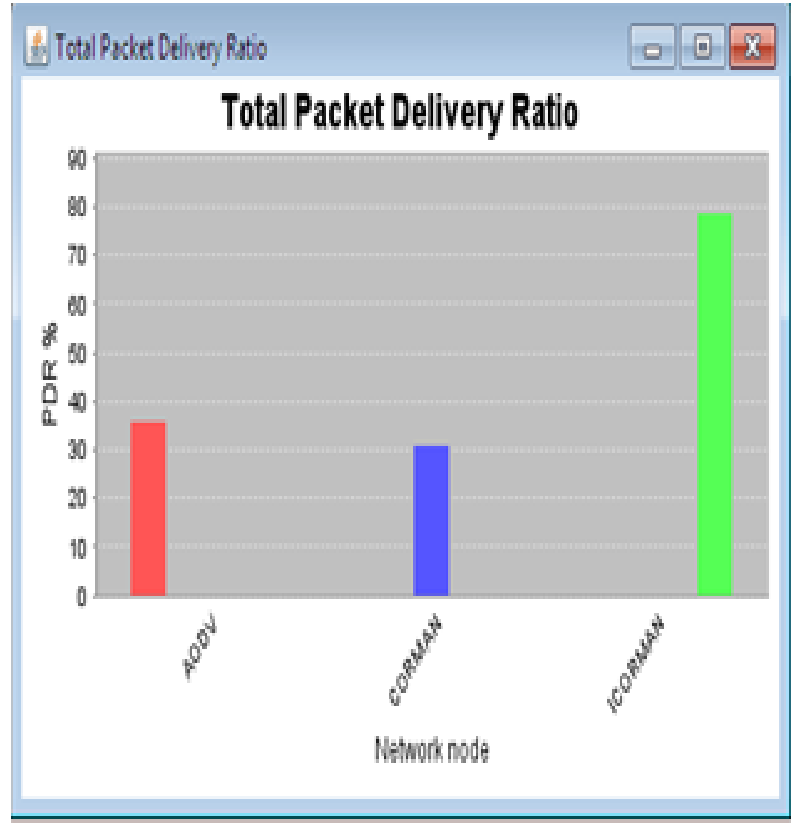

Figure 4: Total packet delivery ratio vs. network node

Above figure 4 indicates the total packet delivery ratio vs. network node for all the three protocols. Total packet delivery ratio is nothing but the ratio of total delivery of the packets by each protocol. As graph shows for AODV, total PDR is 35\%. CORMAN total PDR is $30 \%$. And ICORMAN total PDR is too high i.e. $79 \%$.

At the end it is observed that total PDR of AODV protocol is very less. CORMAN shows good total PDR. But ICORMAN shows better total PDR than both AODV and CORMAN routing protocol.

\subsection{Throughput vs. network node}

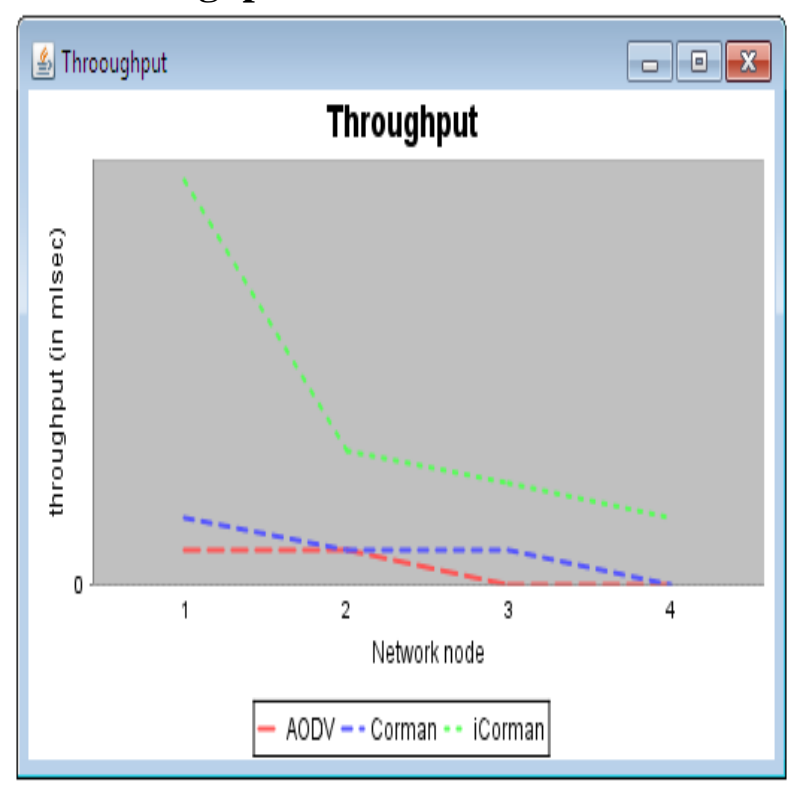

Figure 5: Throughput vs. network node

Above figure 5 indicates the throughput vs. network node. From graph it is cleared that using AODV routing protocol throughput of network performance is 0.01 for second and third node when message is transferred from first node in the network. And it is 0.0 for remaining two nodes. For the same network operation using CORMAN routing protocol it is 0.02 for first node and for node two and node three it is 0.01 . Node four shows 0.0 throughputs for the same network operation. For the same network operation using ICORMAN, second node throughput is 0.12 , for node three it is 0.04 , for node four it is 0.03 and for last node throughput is 0.02 .

At the end it is observed that overall throughput get increase using ICORMAN. And it is less using CORMAN routing protocol and very less using AODV routing protocol.

Final analysis for the PDR, end to end delay, total PDR and throughput performance for ICORMAN, CORMAN and AODV routing protocols shows ICORMAN routing protocol outperforming the existing AODV as well as CORMAN routing protocol. ICORMAN increases the performance of MANET in terms of PDR, throughput, end to end delay and total PDR as compared to AODV and CORMAN routing protocols.

\section{CONCLUSION}

As routing protocol is the key for MANET communications and decides the performance of MANET, designing of efficient routing protocol is the challenging task. This paper addresses the problem of optimal channel allocation by proper utilization of available bandwidth as well as use of efficient channel reuse technique with the aim of improving the performance of MANET. ICORMAN is the new routing protocol in MANET which is the extended version of CORMAN using ACO based efficient channel reuse method. ACO select path with minimum cost and makes the better utilization of channel by making proper utilization of available bandwidth of channel \& improves the routing performance of MANET than AODV as well as CORMAN routing protocol. Simply, ICORMAN outperforms the AODV and CORMAN routing protocols in terms of PDR, end to end delay and throughput.

\section{REFERENCES}

[1] Chlamtac. I, M. Conti, and J.-N. Liu, "Mobile Ad hoc Networking: Imperatives and Challenges," Ad Hoc Networks, vol. 1, no. 1, pp. 13-64, July 2003.

[2] Elizabeth M. Royer, "A Review of Current Routing Protocols for Ad Hoc Mobile Wireless Networks Elizabeth", in 1070-9916/99/\$10.00 @ 1999 IEEE.

[3] Jeroen Hoebeke, Ingrid Moerman, Bart Dhoedt and Piet Demeester," An overview of mobile ad-hoc networks: Applications and challenges".

[4] Donatas Sumyla,"Mobile ad-hoc networks".

[5] Magnus Frodigh, per Johansson and Peter Larsson, "Wireless ad-hoc networking- The art of networking without network".

[6] Kristoffer Karlsson, Billy Ho, "Ad hoc networks: Overview, applications and routing issues".

[7] Zehua Wang, Yuanzhu Chen, Cheng Li, "CORMAN: A Novel Cooperative Opportunistic Routing Scheme in Mobile Ad Hoc Networks" in Communications, IEEE Journal on vol. 30, pp. 289-296, February 2012.

[8] Wang. Z, C. Li, and Y. Chen, "PSR: Proactive Source Routing in Mobile Ad Hoc Networks," in Proc. 2011 IEEE Conference on Global Telecommunications (GLOBECOM), Houston, TX USA, December 2011. 
[9] A. P. Engelbrecht. Fundamentals of Computational Swarm Intelligence, John Wiley \& Sons, UK, 2005.

[10] M. Darigo, T. Stutzle, 'Ant Colony Optimization', MIT Press, Landon, 2004.

[11] Fengji Ye, Su Yi and Biplab Sikdar, "Improving Spatial Reuse of IEEE 802.11 Based Ad Hoc Networks".
[12] Deepender Dhull, Swati Dhull, “An Improved Ant Colony Optimization (IACO) Based Multicasting in MANET in International Journal of Inventive Engineering and Sciences (IJIES) ISSN: 2319-9598, Volume-1, Issue-3, February 2013.

[13] Mahboobeh Parsapoor, Urban Bilstrup,'InterfrenceAware Clustering Algorithms for Mobile ad hoc Network Ant Colony optimization-based Algorithm". 